\title{
Allergic rhinitis, rather than asthma, might be associated with dental caries, periodontitis, and other oral diseases in adults
}

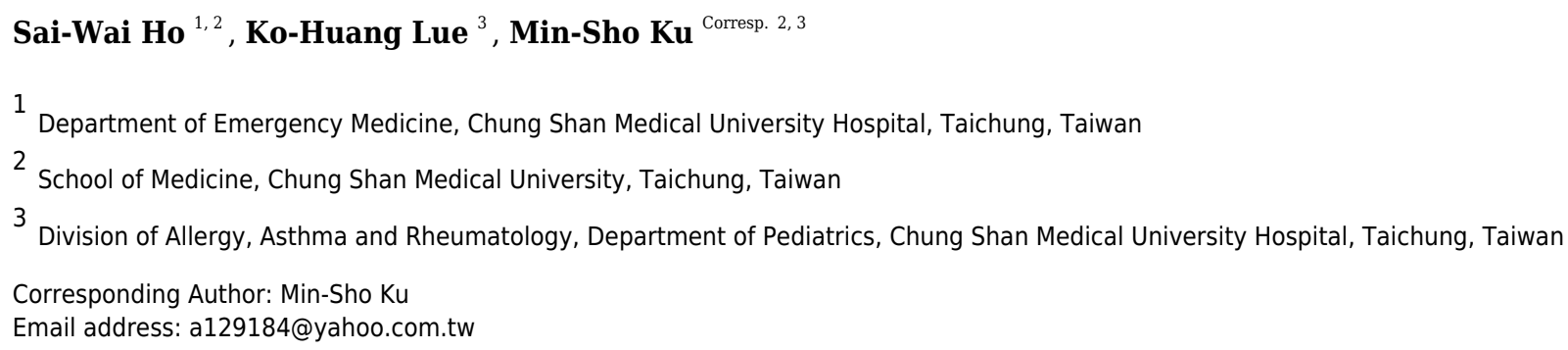

Background. The association between asthma (AS), allergic rhinitis (AR) and oral diseases remains inconclusive in adults. AS and AR often coexist. However, studies that investigate AS, AR together and their association with oral diseases are scarce. Methods. Data from 22,898 men and 28,541 women, aged 21 to 25 years, were collected from a national database in Taiwan. Five common oral diseases: dental caries, periodontitis, pulpitis, gingivitis, and stomatitis/aphthae were studied. Differences in the incidence of the five oral diseases in AR vs. non-AR, and AS vs. non-AS groups were compared. The incidence of the five oral diseases in men/ women, urban/country citizen, and high/ low income groups was studied. The frequencies of clinical visits and impact of topical steroid use between the groups were also studied. The confounding factors included sex, socioeconomic status, urbanization, dentofacial anomalies, disease of salivary flow, diabetes mellitus, and esophageal reflux. Results. The incidence and the frequencies of clinical visits for all five oral diseases were higher in those with AR than in the non-AR group after adjusting for confounding factors and AS. Similar observation was made for the AS group, without adjusting for AR. However, if AR was included for adjustment, no relationship was found between AS and oral diseases. In the AR group, those with higher incomes, and country residents had a high risk of developing oral disease. Intranasal steroids, rather than inhaled steroids, were also associated with oral diseases.

Conclusion. AR, rather than AS, may be associated with oral diseases in young adults. 
1 Allergic rhinitis, rather than asthma, might be associated

2 with dental caries, periodontitis, and other oral diseases

3 in adults

4 Sai-Wai Ho ${ }^{1,2}$, Ko-Huang Lue ${ }^{3}$, Min-Sho $\mathrm{Ku}^{1,3}$

5 1. School of Medicine, Chung Shan Medical University, Taichung, Taiwan.

6 2. Department of Emergency Medicine, Chung Shan Medical University Hospital, Taichung,

7 Taiwan.

8 3. Division of Allergy, Asthma and Rheumatology, Department of pediatrics, Chung Shan

9 Medical University Hospital, Taichung, Taiwan.

10

11

Corresponding Author:

12 Min-Sho $\mathrm{Ku}^{1,3}$

13 110, Section 1, Chien-Kuo North Road, Taichung, 406, Taiwan.

14 Email address: a129184@yahoo.com.tw

15

16

17

18

19

20

21

22

23

24

25

26

27

28

29

30

31

32

33

34

35

36

37 


\section{Abstract}

41 Background. The association between asthma (AS), allergic rhinitis (AR) and oral diseases remains 42 inconclusive in adults. AS and AR often coexist. However, studies that investigate AS, AR together 43 and their association with oral diseases are scarce.

44 Methods. Data from 22,898 men and 28,541 women, aged 21 to 25 years, were collected from a 45 national database in Taiwan. Five common oral diseases: dental caries, periodontitis, pulpitis, gingivitis, and stomatitis/aphthae were studied. Differences in the incidence of the five oral diseases in AR vs. non-AR, and AS vs. non-AS groups were compared. The incidence of the five oral diseases in men/ women, urban/country citizen, and high/ low income groups was studied. The frequencies of clinical visits and impact of topical steroid use between the groups were also studied. The confounding factors included sex, socioeconomic status, urbanization, dentofacial anomalies, disease of salivary flow, diabetes mellitus, and esophageal reflux.

Results. The incidence and the frequencies of clinical visits for all five oral diseases were higher in those with AR than in the non-AR group after adjusting for confounding factors and AS. Similar observation was made for the AS group, without adjusting for AR. However, if AR was included for adjustment, no relationship was found between AS and oral diseases. In the AR group, those with higher incomes, and country residents had a high risk of developing oral disease. Intranasal steroids, rather than inhaled steroids, were also associated with oral diseases.

Conclusion. AR, rather than AS, may be associated with oral diseases in young adults. 


\section{Introduction}

65 Dental caries, periodontitis, pulpitis, gingivitis, and stomatitis/aphthae are common oral diseases

66 (Frencken et al 2017), which have a negative impact on quality of life and work performance. They

67 also lead to increased medical costs. Understanding the risk factors for these conditions may help in

68 their prevention and treatment. The oral diseases are multi-factorial diseases. Some risk factors include

69 oral pathogens, dry mouth, smoking habit, sugar consumption, and systemic diseases (Hunter et al 1988;

$70 \quad$ Yousef et al 2014; Roa et al 2018).

71 The association between AS, caries (Alavaikko et al 2011) and periodontitis/gingivitis

72 (Moraschini et al 2018); and between AR, caries (Wongkamhaeng et al 2014) and periodontitis

73 (Bakhshaee et al 2017) have been reported. However, the underlying mechanisms is different. Salivary

74 micro-flora change due to mouth breathing (Mummolo et al 2018). The effect of Interleukin (IL)-12

75 (Ping et al 2015) has been reported in AR subjects. Decreased IgA levels in gingival tissue have been

76 reported in AS subjects (Ostergaard 1997). Oral micro-flora (Sachs et al 1993; Wongkamhaeng et al

77 2014) and drug prescription (Tootla et al 2004; Elad et al 2006) are two factors that influence oral

78 diseases, and are different between AS and AR.

79

AR and AS often coexist. They are linked by epidemiologic, histologic, physiologic, and

80

81

82

83

84

85

86

87 immunopathologic characteristics, and also by a common therapeutic approach (Simons 1999). AS has been reported to be associated with both oral diseases (Wongkamhaeng et al 2014, Bakhshaee et al 2017) and AR. Compare to non-AR subjects, the frequencies of AS were higher in AR subjects (Simons 1999). Therefore, we speculated that AS might be a confounder while studying the relationship between AR and oral diseases. AR has also been associated with oral diseases (Alavaikko et al 2011, Moraschini et al 2018) and AS. Compare to non-AS subjects, the frequencies of AR were higher in AS subjects (Simons 1999). Therefore, we speculated that AR might be a confounder while studying the relationship between AS and oral diseases. Our previous study found that in children, AR

Peer] reviewing PDF | (2019:04:36547:2:1:CHECK 28 Jul 2019) 
88

89

90

91

92

93

94

95

96

97

98

99

100

101

102

103

104

105

106

107

108

109

110

111

rather than AS, is a risk factor for dental caries (Chuang et al 2018). The confounder AR, brings the relationship between AS and caries into question in children. However, AS and AR, respectively, were not considered as confounders in the previous study on adults.

Studies on the association of AR with caries and periodontitis are few, and they show conflicting results (Wongkamhaeng et al 2014; Hung SH et al 2016; Bakhshaee et al 2017; Kim et al 2018). The association between AS and pulpitis and stomatitis; and between AR and pulpitis, gingivitis, and stomatitis have scarcely been studied. We performed a large population-based research study using the National Health Insurance Research Database (NHIRD) in Taiwan to establish the relationship among the five oral diseases (caries, periodontitis, pulpitis, gingivitis, and stomatitis/aphthae) and AS and AR in young adults. AR was adjusted for when studying the association between AS and oral disease, so we aimed to determine whether the association was induced by AR.

\section{Materials \& Methods}

\section{Database and data collection}

The NHIRD was created by the National Health Research Institute (NHRI) in Taiwan

(Department of health, E. Y. 1998; Cheng 2003). The NHRI randomly sampled a representative database of 1 million subjects in 2010 through systematic sampling, and this sample served as our data source. The database provided information on patient identification, birth date, sex, diagnostic codes from the International Statistical Classification of Diseases and Related Health Problems (ICD)-9-CM, prescription drugs, medical care facilities, and other parameters. This medical information for each subject can be traced forward from year 1985 and backward to 2013.

\section{Criteria for AS, AR and oral diseases}

Subjects born between 1985 and 1988 were selected randomly from the NHIRD. Their claims' data in the age group of 21 to 25 years were analyzed between 2005 and 2013 . The subjects were 
112 divided into the groups: AR and non-AR; and AS and non-AS. The criteria for AR and AS included at 113 least two diagnoses of AR (ICD-9-CM diagnostic code 477) or AS (ICD-9-CM diagnostic code 493) in 114 five years between the ages of 21 and 25 years. Those with disease durations of AR and AS <180 days 115 were excluded because AR and AS are chronic diseases. Five major oral diseases were selected for the 116 study: dental caries (code 521.0), periodontitis (codes 523.3 and 523.4), pulpitis (code 522.0), gingivitis 117 (codes 523.0 and 523.1), and stomatitis (code 528.0)/oral aphthae (code 528.2).

\section{Incidence of oral diseases}

The subjects were followed up for five years (age 21-25 years old). Incidence of the five oral diseases in the AR versus non-AR groups and AS versus non-AS groups, was compared. More than one diagnosis of each oral disease recorded in the study period was defined as having the individual

disease. Incidence of the five oral diseases for AR versus non-AR groups was compared using different demographic characteristics, which included male/ female, urban/ country resident, and high/ low income. The magnitude of the relative risk (RRs) of oral diseases in each character was compared between AR and non-AR subjects.

\section{Clinical visit times for oral diseases}

During the five years follow up, the mean clinical visit times for the five oral diseases in various groups were compared. Mean clinical visit times for three dental treatment methods: dental restoration, endodontics, and periodontitis treatment (surgical and non-surgical) were also compared to investigate the reason for clinical visit.

\section{Influence of use of inhaled steroids for AS and intranasal steroids for AR}

AR subjects were divided into intranasal steroid group (who had used intranasal steroids during the 
137 period). AS subjects were divided into inhaled steroid group (who had used inhaled steroids during the

138 study period) and non-inhaled steroid group (who had never used inhaled steroids during the study

139 period). Clinical visit times and treatments for oral disease were compared between the groups.

140 Anatomical Therapeutic Chemical code R01AD was used for intranasal steroids, and R03AK and

141 R03BA for inhaled steroids.

142

143 Determining confounding factors

144 Factors that influence oral health include sex, socioeconomic status, urbanization, dentofacial 145 anomalies (ICD-9-CM code 524), salivary flow diseases (ICD-9-CM codes 527 and 710.2), diabetes

146 mellitus (DM) (ICD-9-CM code 250) and esophageal reflux (ICD-9-CM codes 530.11 and 530.81)

147 (Hunter et al 1988; Yousef et al 2014; Roa et al 2018). These factors were considered as risk factors

148 and were adjusted for. AS was used as the confounding factor when investigating the association

149 between AR and oral diseases, and AR was used as the confounding factor when investigating the 150 association between AS and oral diseases.

151 Socioeconomic status was defined according to the occupation, wherein, the subjects were grouped

152 into high-income (teacher or public official, company employee) and low-income (peasants, fishermen, 153 others, low income or no fixed job) groups. Based on Liu's report (Liu et al. 2006), urbanization was 154 grouped into seven levels: levels 1-2 for urban residents, and levels 3-7 for country residents.

156 Statistical analysis

157 All analyses were performed using SAS version 9.1 for Windows (SAS Inc., Cary, NC, USA) and 158 PASW Statistics 18 (IBM, Armonk, NY, USA). For the five oral diseases, we assessed model 159 calibration with the Hosmer-Lemeshow goodness of fit test. The model was regarded as no goodness 160 of fit in case of $\mathrm{p}<0.05$. Mantel-Haenszel chi-square tests stratified for different characteristics (sex, 161 urbanization and income) were used to examine the potential effect modifiers among oral diseases and 
162 AR. Chi-square test was used to compare the frequencies of characteristics between groups. Modified

163 Poisson regression analysis was used to obtain RRs for the incidence of oral diseases between the 164 groups. Negative binominal regression was performed for assessing differences in the frequencies of 165 oral disease and clinical treatment visits between the groups. Crude $\mathrm{p}$ values and adjusted $\mathrm{p}$ value for 166 confounders (sex, socioeconomic status, urbanization, dentofacial anomalies, diseases of salivary flow, 167 DM, esophageal reflux, AS, AR) were calculated. Two-sided p values of $<0.05$ were defined as 168 significant.

169 Results

\section{Demographic data, goodness of fit and potential effect modifiers}

Demographic data are presented in Table 1. Total 51,439 subjects were recruited, of which 22,898 (44.5\%) were men and 28,541 (55.5\%) were women. Of these subjects, 7,884 (15.3\%) fell into the AR group [3,247 (14.2\%) men and 4,637 (16.2\%) women], whereas, 1,232 (2.4\%) fell into the AS group [569 (2.5\%) men and $663(2.3 \%)$ women]. Hosmer-Lemeshow test $\mathrm{p}$ values for the five oral diseases were as follows: caries (0.059), periodontitis (0.038), pulpitis (0.565), gingivitis $(0.303)$, and aphthae/stomatitis (0.074). Sex, urbanization and income was the potential effect modifiers among caries, periodontitis and AR. Sex, and income was the potential effect modifiers among gingivitis and AR. Sex was the potential effect modifiers among gingivitis and AR.

179

180

181

182

183

184

185

\section{Incidence of oral diseases}

The results for incidence of oral diseases are presented in Table 2. Incidence of the five oral diseases was higher in AR subjects as compared to non-AR subjects, and this difference was statistically significant. The statistically significant difference was still noted if AS was added for adjustment. The incidence of caries, periodontitis, gingivitis, and aphthae/stomatitis was higher in AS 
subjects as compared to non-AS subjects, and this difference was statistically significant. However, if

187 AR was added for adjustment, all the differences became non-significant. The incidence of oral

188 diseases between AR and non-AR subjects, considering the demographic characteristics, is presented in

Table 3. Except for pulpitis in men, urban citizens and subjects with low income the incidence was all significantly higher in AR subjects.

To evaluate the magnitude of AR's influence on oral diseases, the RRs data for each demographic

character and each oral disease was compared (Table 3). RRs (AR vs. non-AR) for dental caries was

higher (1.17) in men than women (1.09). RRs (AR vs non-AR) of periodontitis, gingivitis and

aphthae/periodontitis was higher in men than women. RRs (AR vs non-AR) of the five diseases was

higher in AR country residents and AR subjects with high incomes than AR urban residents and AR

subjects with low incomes.

197

\section{Clinical visits for oral diseases and treatment}

Mean clinical visit times for the five oral diseases and three treatments were significantly higher in

AR subjects during the study period (Table 4). The most common reason for clinical visit was

restoration, followed by periodontitis treatment and endodontics (Table 4). The statistically significant

difference was still noted if AS was added for adjustment. Except for endodontics treatment, mean

clinical visit times for five oral diseases and two treatments were significantly higher in AS subjects;

however, if AR was added for adjustment, the differences became non-significant.

\section{Association between inhaled steroids, intranasal steroids and oral diseases}

The results for this association are presented in Table 5. In AR subjects, mean clinical visit times

for the oral diseases except pulpitis, and treatment except endidontics, were significantly higher in those using intranasal steroids; and the statistically significant difference was still noted after 
210 adjustment for AS. In AS subjects, there was no significant association between mean clinical visit

211 times for oral diseases and treatments of those who inhaled steroids and those who did not.

\section{Discussion}

213 Our study found that AR was associated with dental caries, periodontitis, pulpitis, gingivitis and 214 aphthae/stomatitis. However, we did not find any association between AS and the five oral diseases 215 after adjusting for AR. The clinical visit times for caries, periodontitis, gingivitis, aphthae/stomatitis, 216 periodontitis, and restoration treatment times were higher in AR subjects using intranasal steroids. Also, 217 in AR group, men, country residents and those with high income had higher incidence of oral diseases. 218 Studies on the relationship between AR and caries reveal conflicting results. Some studies found a 219 positive association (Bakhshaee et al 2017; Chuang et al 2018), while others found no association 220 (Tanaka et al 2008; Wongkamhaeng et al 2014). No studies have been reported in adults. The relationship between AR and periodontitis is inconclusive, because both positive (Hung SH et al 2016) and inverse (Kim et al 2018) associations have been reported. To the best of our knowledge, an association between AR and gingivitis, pulpitis, and stomatitis/aphthae has not been reported previously.

Our study provides evidence that AR is associated with five oral diseases (caries, periodontitis, pulpitis, gingivitis, and stomatitis/aphthae). The increased prevalence rate may imply that AR influences development of oral diseases, while increased clinical visit times mean that AR might increase the severity of oral diseases. Restoration and endodontics treatment are used for caries, periodontitis, and pulpitis; and increased treatment visit times in AR subjects make the association more certain. Among the AR subjects, men, country residents and those with high income had higher

231 RRs for oral diseases. This might suggest that men who are country residents with a high income 232 should pay more attention to their oral health if they also have AR. 
There have been more studies investigating the association between AS and caries, periodontitis

234

235

236

237

238

239

240

241

242

243

244

245

246

247

248

and gingivitis, than on the association with AR. Many studies have reported a positive association between AS and caries (Alavaikko et al 2011), and periodontitis and gingivitis (Moraschini et al 2018). However, most of the studies did not adjust for AR, which might be an important confounder. In our study, before adjusting for AR, incidence for four oral diseases (Table 1), clinical visit times for five oral disease and three oral treatments was significantly higher in AS group than the non-AS group. However, after adjusting for AR, the differences became non-significant. So, we concluded that AS is not associated with oral disease. AR might be a confounding factor associated with both AS and oral diseases. Inhaled steroids have been reported to increase the incidence of caries, periodontitis, gingivitis, and oral ulcers, with change in oral $\mathrm{pH}$, local deposition of steroids in the oral cavity, and their effect on oral mucosa (Alavaikko et al 2011; Bozejac et al 2017). The study of intranasal steroids and oral diseases is limited. We speculate that local deposition of steroids due to postnasal dripping might induce the same oral diseases that inhaled steroids do. In our study, three oral diseases and two oral treatment visit times were significantly associated with the use of intranasal steroids, but no association was noted between inhaled steroids and oral diseases (Table 4). Intranasal steroids are more expensive than inhaled steroids in Taiwan, so these drugs are prescribed if patients have more severe AR symptoms. More studies are needed to explain our findings.

The results of several studies could explain the association between AS, AR, and oral diseases. At the mechanistic level, a decreased saliva secretion rate has been reported in both AS (Lenander et al 1998) and AR (Elad et al 2006) subjects. Dehydration of the gingiva due to mouth breathing in AR subjects may also be a contributing factor for gingivitis. At the pharmacological level, antihistamines (Elad et al 2006) and inhaled $\beta_{2}$-agonists (Tootla et al 2004) could decrease salivary flow. At the microbiological level, AS (Sachs et al 1993) and AR (Wongkamhaeng et al 2014) subjects have been found to have different oral micro-flora than the control subjects. At the immunological level, decreased IgA levels in gingival tissue have been reported in allergic disease (Ostergaard 1997), and 
258 IgA is a first-line defense immunoglobulin for mucosa, and plays a role in restricting periodontal 259 disease. Another study indicated that IL-12 is associated with AR (Ping et al 2015); IL-12 may also be 260 related to the pathogenesis of periodontal disease (Tsai IS et al 2005).

261 Our study has several strengths. First, other studies have assessed AR or AS from past history, 262 rather than being an existing condition at the time of study. AR and AS subjects in our study were in 263 the active stage of allergic disease with more than two diagnoses within the study period. Studying the 264 association between oral diseases and AR and AS during the active stage of allergic diseases is more 265 pertinent. Second, by knowing the frequencies of clinical visits and treatment times, we could 266 understand the influence of allergic disease on the severity of oral diseases, medical treatment, and 267 medical costs.

268 There are limitations to our study. First, there are no laboratory data such as oral pathogenic 269 bacteria, IgA in oral samples/saliva and mucus. Second, there are no data on smoking habit, sugar 270 consumption, engagement with the healthcare system, personal oral hygiene, and oral drugs. Third, the

271 Hosmer-Lemeshow test was used to evaluate the model's calibration. Caries, pulpitis, gingivitis and 272 aphthae/stomatitis showed a good fit, but periodontitis did not. Future study for the association between 273 AR and periodontitis is necessary.

\section{Conclusions}

The present study provides evidence that AR might be associated with caries, periodontitis, pulpitis, gingivitis, and stomatitis/aphthae in young adults. Based on increased clinical visit times for the five diseases, it can be speculated that AR also increases the severity of these five oral diseases. Contrary to findings in other studies, there is no association between AS and the five oral diseases. Any association between AS and oral diseases previously found might be due to AR.

Men who live in the countryside and have a high income should pay more attention to oral 
282 demographic categories. Intranasal steroids used in AR, rather than inhaled steroids used in AS, are 283 associated with the development of oral diseases.

284 In order to prevent and treat oral diseases, simultaneous treatment of AR is important. Considering 285 the effects of AR in the elucidation of the etiology of oral diseases is a new direction of study. However 286 more studies are required to ensure whether AR is the cause of oral diseases. More biological research 287 and more epidemiological data on the relationship between AR, AS and oral diseases are necessary in 288 order to gain clarity.

289

\section{Acknowledgements}

291 None

292

293

294

295

296

297

298

299

300

301

302

303

304

305

306 


\title{
References
}

\author{
Alavaikko S, Jaakkola MS, Tjäderhane L, Jaakkola JJ. 2011.
}

Asthma and Caries: A Systematic Review and Meta-Analysis. American Journal of Epidemiology 174:631-641. DOI: 10.1093/aje/kwr129.

\section{Bakhshaee M, Ashtiani SJ, Hossainzadeh M, Sehatbakhsh S, Najafi MN, Salehi M. 2017.} Allergic rhinitis and dental caries in preschool children. Dental Research Journal 14:376-381. PMID:29238375.

Bozejac BV; Stojsin I; uric M; Zvezdin B; Brkanic T; Budisin E; Vukoje K; Secen N. 2017. Impact of inhalation therapy on the incidence of carious lesions in patients with asthma and COPD. Journal of Applied Oral Science 25:506-514. DOI: 10.1590/1678-7757-2016-0147.

\section{Chuang CY, Sun HL, Ku MS. 2018.}

Allergic rhinitis, rather than asthma, is a risk factor for dental caries. Clinical Otolaryngology 43:131-136. DOI: 10.1111/coa.12912.

\section{Cheng TM. 2003.}

Taiwan's new national health insurance program: genesis and experience so far. Health Affairs 22:61-76. DOI:10.1377/hlthaff.22.3.61.

\section{Department of health, E. Y. 1998.}

National Health Insurance Evaluation and Projections. Taiwan: 1998; DoH,1.

\section{Elad S, Heisler S, Shalit M. 2006.}

Saliva secretion in patients with allergic rhinitis. International Archived od Allergy and Immunology 141:276-280. DOI:10.1159/000095297.

Frencken JE, Sharma P, Stenhouse L, Green D, Laverty D, Dietrich T. 2017

Global epidemiology of dental caries and severe periodontitis - a comprehensive review. Journal of Clinical Periodontology S18:S94-S105. DOI: 10.1111/jcpe.12677.

\section{Hunter PB. 1988}

Risk factors in dental caries. Int Dent J. 38:211-217. PMID: 3063664.

Hung SH, Tsai MC, Lin HC, Chung SD. 2016.

Allergic rhinitis is associated with periodontitis: a population-based study. Journal of periodontology 87:749-755. DOI: 10.1902/jop.2016.150539.

\section{Kim EJ, Choi YK. 2018.}

Allergic rhinitis and periodontitis among Korean adults: results from a nationwide population-based study (2013-2015). BMC Ear, Nose and Throat Disorders 18: 12. DOI: $10.1186 / \mathrm{s} 12901-018-0062-3$.

\section{Liu CY, Hung YT, Chuang YL, Chen YJ, Weng WS, Liu JS. 2006.} Incorporating development stratification of Taiwan townships into sampling design of large scale health interview survey. Journal of Health Management 4:1-22.

\section{Lenander-Lumikari M, Laurikainen K, Kuusisto P, Vilja P. 1998.}

Stimulated salivary flow rate and composition in asthmatic and non-asthmatic adults. Archives of Oral Biology 43:151-156. PMID: 9602294

\section{Mummolo S, Nota A, Caruso S, Quinzi V, Marchetti E, Marzo G. 2018.}

Salivary Markers and Microbial Flora in Mouth Breathing Late Adolescents. BioMed Research International 5:8687608. DOI: 10.1155/2018/8687608. 
Moraschini V, Calasans-Maia JA, Calasans-Maia MD. 2018. Association between asthma and periodontal disease: A systematic review and meta-analysis. Journal of periodontology 89:440-455. DOI: 10.1902/jop.2017.170363. pathologica et microbiologica Scandinavica. Section C. 85:187-195. PMID:327753. the Chinese Han population. Journal of the European Federation of Oto-Rhino-Laryngological Societies 272:889-893. DOI: 10.1007/s00405-014-3204-2.

Roa I, Del Sol. M. 2018.

Obesity, salivary glands and oral pathology. Colomb Med (Cali). 49:280-287.

DOI:10.25100/cm.v49i3.3919.

\section{Simons FE. 1999.}

Allergic rhinobronchitis: the asthma-allergic rhinitis link. Journal of Allergyand Clinical Immunology 104:534-540. PMID:10482824

Sachs AP, van der Waaij D, Groenier KH, Koëter GH, Schiphuis J. 1993. oropharyngeal microorganisms in outpatients with asthma or chronic obstructive pulmonary disease. The American Review of Respiratory Disease 148:1302-1307. Journal of Asthma 45:795-799. DOI: 10.1080/02770900802252119.

\section{Tsai IS, Tsai CC, Ho YP, Ho KY, Wu YM, Hung CC. 2005.} Interleukin-12 and interleukin-16 in periodontal disease. Cytokine 31:34-40. DOI:10.1016/j.cyto.2005.02.007.

\section{Tootla R, Toumba KJ, Duggal MS. 2004.}

An evaluation of the acidogenic potential of asthma inhalers. Archives of Oral Biology 49:275-283. DOI:10.1016/j.archoralbio.2003.11.006. DOI: $10.1155 / 2014 / 182513$. 
Table $\mathbf{1}$ (on next page)

characteristics of AR vs non-AR and AS vs non-AS

characteristics of AR vs non-AR and AS vs non-AS 
1 Table 1. Characteristics of AR vs non-AR and AS vs non-AS

\begin{tabular}{|c|c|c|c|c|c|c|}
\hline & AR & Non-AR & Crude $p$-value & AS & Non-AS & Crude $p$ - \\
\hline & $\begin{array}{c}n=7,884 \\
(15.3 \%)\end{array}$ & $\begin{array}{c}\mathrm{n}=43,555 \\
(84.7 \%)\end{array}$ & & $\begin{array}{c}\mathrm{n}=1,232 \\
(2.4 \%)\end{array}$ & $\begin{array}{c}\mathrm{n}=50,207 \\
(97.6 \%)\end{array}$ & \\
\hline Sex & & & & & & \\
\hline Male & $3,247(41.2 \%)$ & $19,651(45.1 \%)$ & $<0.001$ & $569(46.2 \%)$ & $22,329(44.5 \%)$ & 0.2 \\
\hline Female & $4,637(58.8 \%)$ & $23,904(54.9 \%)$ & & $663(53.8 \%)$ & $27,878(55.5 \%)$ & \\
\hline Socioeconomic st & & & & & & \\
\hline High income & $4,415(56.0 \%)$ & $24,361(55.9 \%)$ & 0.911 & $673(54.6 \%)$ & $28,103(56.0 \%)$ & 0.3 \\
\hline Low income & $3,469(44.0 \%)$ & $19,194(44.1 \%)$ & & $559(45.4 \%)$ & $22,104(44.0 \%)$ & \\
\hline Urbanization & & & & & & \\
\hline Urban & $4,725(59.9 \%)$ & $25,230(57.9 \%)$ & $<0.001$ & $798(64.8 \%)$ & $29,157(58.1 \%)$ & $<0.00$ \\
\hline Country & $3,159(40.1 \%)$ & $18,325(42.1 \%)$ & & $434(35.2 \%)$ & $21,050(41.9 \%)$ & \\
\hline Dentofacial anomalies & $1,466(18.6 \%)$ & $5,748(13.2 \%)$ & $<0.001$ & $191(15.5 \%)$ & $7,023(14.0 \%)$ & 0.130 \\
\hline Disease of salivary flow & $92(1.2 \%)$ & $240(0.6 \%)$ & $<0.001$ & $12(1.0 \%)$ & $320(0.6 \%)$ & 0.14 \\
\hline $\mathrm{DM}$ & $50(0.6 \%)$ & $198(0.5 \%)$ & 0.034 & $13(1.1 \%)$ & $235(0.5 \%)$ & 0.00 \\
\hline Esophageal reflux & $295(3.7 \%)$ & $700(1.6 \%)$ & $<0.001$ & $65(5.3 \%)$ & $930(1.9 \%)$ & $<0.0$ \\
\hline
\end{tabular}

2 Chi-square test was used to compare the frequencies of characteristics between groups.

3 Abbreviations: AS: asthma, AR: allergic rhinitis, DM: diabetes mellitus

4

5

6

7

8

9

10

11

12

13

14

15

16

17

18 


\section{Table 2 (on next page)}

The incidence of oral disease in AR vs non-AR and AS vs non-AS

The incidence of oral disease in AR vs non-AR and AS vs non-AS 
1 Table 2: The incidence of oral disease in AR vs. non-AR and AS vs. non-AS subjects

\begin{tabular}{|l|c|c|c|c|c|}
\hline & AR & Non-AR & $p$-value (a) & $p$-value (b) & $p$-value [RRs (95\% CI)] (c) \\
\hline & $\mathrm{n}=7,884$ & $\mathrm{n}=43,555$ & & & \\
\hline Caries & $79.7 \%$ & $69.1 \%$ & $<0.001$ & $<0.001$ & $<0.001[1.12(1.11-1.14)]$ \\
\hline Periodontitis & $71.5 \%$ & $59.9 \%$ & $<0.001$ & $<0.001$ & $<0.001[1.16(1.14-1.18)]$ \\
\hline Pulpitis & $25.7 \%$ & $23.2 \%$ & $<0.001$ & $<0.001$ & $<0.001[1.08(1.04-1.13)]$ \\
\hline Gingivitis & $60.5 \%$ & $49.5 \%$ & $<0.001$ & $<0.001$ & $<0.001[1.17(1.15-1.20)]$ \\
\hline Aphthae/stomatitis & $29.7 \%$ & $19.8 \%$ & $<0.001$ & $<0.001$ & $<0.001[1.39(1.34-1.45)]$ \\
\hline & $\mathrm{AS}$ & Non-AS & $p$-value (a) & $p$-value (b) & $p$-value [RRs (95\% CI)] (d) \\
\hline & $\mathrm{n}=1,232$ & $\mathrm{n}=50,207$ & & & \\
\hline Caries & $76.8 \%$ & $70.6 \%$ & $<0.001$ & $<0.001$ & $0.109[1.03(0.99-1.06)]$ \\
\hline Periodontitis & $67.8 \%$ & $61.5 \%$ & $<0.001$ & $<0.001$ & $0.290[1.02(0.98-1.06)]$ \\
\hline Pulpitis & $25.5 \%$ & $23.5 \%$ & 0.100 & 0.130 & $0.421[1.04(0.94-1.15)]$ \\
\hline Gingivitis & $57.7 \%$ & $51.0 \%$ & $<0.001$ & $<0.001$ & $0.115[1.04(0.99-1.09)]$ \\
\hline Aphthae/stomatitis & $27.2 \%$ & $21.2 \%$ & $<0.001$ & $<0.001$ & $0.104[1.08(0.98-1.18)]$ \\
\hline
\end{tabular}

2 Modified poisson regression was used to calculate the relative risks (RRs) and to adjust the

3 confounders.

4 (a). Crude $p$-value

5 (b). Adjusted by sex, socioeconomic status, urbanization, dentofacial anomalies, disease of

6 salivary flow, DM, esophageal reflux

7 (c). Adjusted by factors (b) plus AS

8 (d). Adjusted by factors (b) plus AR

9 Abbreviations: AS: asthma, AR: allergic rhinitis, CI: confidence interval, DM: diabetes mellitus 


\section{Table 3 (on next page)}

The incidence of oral disease in AR vs non-AR in different characters

The incidence of oral disease in AR vs non-AR in different characters 
Table 3: The incidence of oral disease in AR vs. non-AR, in different characters

\begin{tabular}{|c|c|c|c|c|c|}
\hline & Caries & Periodontitis & Pulpitis & Gingivitis & Aphthae/stomatitis \\
\hline \multicolumn{6}{|l|}{ Male } \\
\hline AR vs non-AR & $74.7 \% ; 62.3 \%$ & $66.2 \% ; 54.1 \%$ & $22.7 \% ; 20.8 \%$ & $54.9 \% ; 43.8 \%$ & $25.2 \% ; 16.1 \%$ \\
\hline RRs (95\% CI) & $1.17(1.15-1.20)$ & $1.19(1.16-1.23)$ & $1.06(0.99-1.14)$ & $1.21(1.17-1.26)$ & $1.46(1.36-1.56)$ \\
\hline$p$-value & $<0.001$ & $<0.001$ & 0.094 & $<0.001$ & $<0.001$ \\
\hline \multicolumn{6}{|l|}{ Female } \\
\hline AR vs non-AR & $83.2 \% ; 74.7 \%$ & $75.3 \% ; 64.6 \%$ & $27.8 \% ; 25.1 \%$ & $64.5 \% ; 54.2 \%$ & $32.9 \% ; 22.9 \%$ \\
\hline RRs (95\% CI) & $1.09(1.08-1.11)$ & $1.14(1.12-1.16)$ & $1.10(1.04-1.16)$ & $1.15(1.12-1.18)$ & $1.36(1.30-1.43)$ \\
\hline$p$-value & $<0.001$ & $<0.001$ & $<0.001$ & $<0.001$ & $<0.001$ \\
\hline \multicolumn{6}{|l|}{ Urban } \\
\hline AR vs non-AR & $79.9 \% ; 70.7 \%$ & $72.1 \% ; 61.9 \%$ & $24.8 \% ; 23.2 \%$ & $60.9 \% ; 51.3 \%$ & $29.1 \% ; 20.0 \%$ \\
\hline RRs (95\% CI) & $1.11(1.09-1.12)$ & $1.14(1.12-1.17)$ & $1.05(0.99-1.11)$ & $1.15(1.12-1.18)$ & $1.37(1.30-1.44)$ \\
\hline$p$-value & $<0.001$ & $<0.001$ & 0.081 & $<0.001$ & $<0.001$ \\
\hline \multicolumn{6}{|l|}{ Country } \\
\hline AR vs non-AR & $79.4 \% ; 66.9 \%$ & $70.7 \% ; 57.3 \%$ & $27.0 \% ; 23.1 \%$ & $60.1 \% ; 47.0 \%$ & $30.6 \% ; 19.9 \%$ \\
\hline RRs $(95 \% \mathrm{CI})$ & $1.15(1.12-1.17)$ & $1.18(1.15-1.21)$ & $1.13(1.06-1.21)$ & $1.22(1.18-1.26)$ & $1.42(1.34-1.51)$ \\
\hline$p$-value & $<0.001$ & $<0.001$ & $<0.001$ & $<0.001$ & $<0.001$ \\
\hline \multicolumn{6}{|l|}{ High income } \\
\hline AR vs non-AR & $80.5 \% ; 68.5 \%$ & $72.4 \% ; 58.5 \%$ & $24.4 \% ; 21.6 \%$ & $61.4 \% ; 50.0 \%$ & $29.5 \% ; 19.6 \%$ \\
\hline RRs $(95 \% \mathrm{CI})$ & $1.14(1.12-1.16)$ & $1.18(1.16-1.21)$ & $1.11(1.05-1.18)$ & $1.18(1.15-1.21)$ & $1.41(1.33-1.48)$ \\
\hline$p$-value & $<0.001$ & $<0.001$ & $<0.001$ & $<0.001$ & $<0.001$ \\
\hline \multicolumn{6}{|l|}{ Low income } \\
\hline AR vs non-AR & $78.7 \% ; 69.9 \%$ & $70.4 \% ; 60.4 \%$ & $27.4 \% ; 25.2 \%$ & $59.4 \% ; 48.9 \%$ & $29.8 \% ; 20.1 \%$ \\
\hline RRs (95\% CI) & $1.09(1.07-1.12)$ & $1.13(1.10-1.16)$ & $1.06(0.99-1.12)$ & $1.17(1.13-1.21)$ & $1.37(1.29-1.46)$ \\
\hline$p$-value & $<0.001$ & $<0.001$ & 0.075 & $<0.001$ & $<0.001$ \\
\hline
\end{tabular}

2 Modified poisson regression was used to calculate the relative risks (RRs) and p-value

3 p-value: adjusted by sex, socioeconomic status, urbanization, dentofacial anomalies, disease of

4 salivary flow, DM, esophageal reflux and AS

5 Abbreviations: AS: asthma, AR: allergic rhinitis, CI: confidence interval, DM: diabetes mellitus

6

7

8

9

10 


\section{Table 4(on next page)}

Mean clinical visit times (over 5 years) for oral disease and treatments, for AR vs. non$A R$; and AS vs. non-AS subjects

Mean clinical visit times (over 5 years) for oral disease and treatments, for AR vs. non-AR; and AS vs. non-AS subjects 
1 Table 4: Mean clinical visit times (over 5 years) for oral disease and treatments, for

2 AR vs. non-AR; and AS vs. non-AS subjects

\begin{tabular}{|l|c|c|c|c|c|c|}
\hline & AR & Non-AR & Ratio & $p$-value (a) & $p$-value (b) & $p$-value [95\% CI] (c) \\
\hline Caries & 3.91 & 3.08 & 1.27 & $<0.001$ & $<0.001$ & $<0.001[1.19-1.27]$ \\
\hline Periodontitis & 2.16 & 1.61 & 1.34 & $<0.001$ & $<0.001$ & $<0.001[1.25-1.33]$ \\
\hline Pulpitis & 0.55 & 0.48 & 1.15 & $<0.001$ & $<0.001$ & $<0.001[1.05-1.18]$ \\
\hline Gingivitis & 1.42 & 1.04 & 1.37 & $<0.001$ & $<0.001$ & $<0.001[1.26-1.35]$ \\
\hline Aphthae/stomatitis & 0.60 & 0.35 & 1.71 & $<0.001$ & $<0.001$ & $<0.001[1.49-1.66]$ \\
\hline Periodontitis treatment & 6.70 & 5.08 & 1.32 & $<0.001$ & $<0.001$ & $<0.001[1.25-1.30]$ \\
\hline Restoration & 7.45 & 6.05 & 1.23 & $<0.001$ & $<0.001$ & $<0.001[1.17-1.23]$ \\
\hline Endodontics & 1.79 & 1.62 & 1.10 & $<0.001$ & 0.004 & $0.009[1.02-1.13]$ \\
\hline & $\mathrm{AS}$ & Non-AS & Ratio & $p$-value (a) & $p$-value (b) & $p$-value [95\% CI] (d) \\
\hline Caries & 3.60 & 3.20 & 1.13 & $<0.001$ & 0.001 & $0.461[0.96-1.10]$ \\
\hline Periodontitis & 1.94 & 1.69 & 1.15 & $<0.001$ & $<0.001$ & $0.541[0.95-1.10]$ \\
\hline Pulpitis & 0.58 & 0.49 & 1.18 & 0.014 & 0.020 & $0.096[0.98-1.29]$ \\
\hline Gingivitis & 1.32 & 1.09 & 1.21 & $<0.001$ & $<0.001$ & $0.095[0.99-1.15]$ \\
\hline Aphthae/stomatitis & 0.52 & 0.39 & 1.33 & $<0.001$ & $<0.001$ & $0.210[0.95-1.25]$ \\
\hline Periodontitis treatment & 6.09 & 5.31 & 1.15 & $<0.001$ & $<0.001$ & $0.374[0.97-10.8]$ \\
\hline Restoration & 7.03 & 6.24 & 1.13 & $<0.001$ & 0.001 & $0.329[0.97-1.11]$ \\
\hline Endodontics & 1.82 & 1.64 & 1.11 & 0.102 & 0.097 & $0.243[0.95-1.22]$ \\
\hline
\end{tabular}

3 Negative binomial regression was used to calculate the frequencies and to adjust the confounders.

4 (a). Crude $p$-value

5 (b). Adjusted by sex, socioeconomic status, urbanization, dentofacial anomalies, disease of

6 salivary flow, DM, esophageal reflux

7 (c). Adjusted by factors (b) plus AS, with 95\% CI

8 (d). Adjusted by factors (b) plus AR, with 95\% CI

9 Abbreviations: AS: asthma, AR: allergic rhinitis, CI: confidence interval, DM: diabetes mellitus 


\section{Table 5 (on next page)}

Mean clinical visit times (over 5 years) for oral disease and treatments for AR subjects who had used intranasal steroids (38.6) vs. those who never had (61.4\%), and AS subjects who had used inhaled steroids (55.4\%) vs. those who never had $(44.6 \%)$

Mean clinical visit times (over 5 years) for oral disease and treatments for AR subjects who had used intranasal steroids (38.6) vs. those who never had (61.4\%), and AS subjects who had used inhaled steroids (55.4\%) vs. those who never had (44.6\%) 
1 Table 5: Mean clinical visit times (over 5 years) for oral disease and treatments for

2 AR subjects who had used intranasal steroids (38.6) vs. those who never had (61.4\%),

3 and AS subjects who had used inhaled steroids (55.4\%) vs. those who never had (44.6\%)

\begin{tabular}{|l|c|c|c|c|c|c|}
\hline AR with nasal steroid & yes & no & Ratio & $p$-value (a) & $p$-value (b) & $p$-value [95\% CI] (c) \\
\hline Caries & 4.18 & 3.74 & 1.12 & $<0.001$ & $<0.001$ & $<0.001[1.07-1.18]$ \\
\hline Periodontitis & 2.28 & 2.09 & 1.09 & $<0.001$ & $<0.001$ & $<0.001[1.04-1.15]$ \\
\hline Pulpitis & 0.57 & 0.54 & 1.06 & 0.279 & 0.257 & $0.281[0.95-1.18]$ \\
\hline Gingivitis & 1.53 & 1.35 & 1.13 & $<0.001$ & $<0.001$ & $<0.001[1.07-1.19]$ \\
\hline Aphthae/stomatitis & 0.64 & 0.57 & 1.12 & 0.021 & 0.032 & $0.035[1.01-1.22]$ \\
\hline Periodontitis treatment & 7.08 & 6.46 & 1.10 & $<0.001$ & $<0.001$ & $<0.001[1.06-1.14]$ \\
\hline Restoration & 7.83 & 7.21 & 1.09 & $<0.001$ & $<0.001$ & $<0.001[1.04-1.14]$ \\
\hline Endodontics & 1.85 & 1.75 & 1.06 & 0.242 & 0.183 & $0.209[0.97-1.17]$ \\
\hline AS with inhaled steroid & yes & no & Ratio & $p$-value (a) & $p$-value (b) & $p$-value [95\% CI] (d) \\
\hline Caries & 3.44 & 3.73 & 0.92 & 0.208 & 0.307 & $0.195[0.81-1.04]$ \\
\hline Periodontitis & 1.99 & 1.90 & 1.05 & 0.489 & 0.564 & $0.752[0.90-1.16]$ \\
\hline Pulpitis & 0.58 & 0.57 & 1.02 & 0.928 & 0.767 & $0.735[0.80-1.38]$ \\
\hline Gingivitis & 1.25 & 1.39 & 0.90 & 0.168 & 0.153 & $0.069[0.76-1.01]$ \\
\hline Aphthae/stomatitis & 0.48 & 0.55 & 0.87 & 0.280 & 0.505 & $0.172[0.65-1.08]$ \\
\hline Periodontitis treatment & 6.06 & 6.12 & 0.99 & 0.830 & 0.805 & $0.411[0.87-1.06]$ \\
\hline Restoration & 6.97 & 7.07 & 0.99 & 0.825 & 0.943 & $0.623[0.86-1.10]$ \\
\hline Endodontics & 1.89 & 1.76 & 1.07 & 0.580 & 0.491 & $0.545[0.85-1.37]$ \\
\hline
\end{tabular}

4 Negative binomial regression was used to calculate the frequencies and to adjust the

5 confounders.

6 (a). Crude $p$-value

7 (b). Adjusted by sex, socioeconomic status, urbanization, dentofacial anomalies, disease of

8 salivary flow, DM, esophageal reflux

9 (c). Adjusted by factors (b) plus AS, with 95\% CI

10 (d). Adjusted by factors (b) plus AR, with 95\% CI

11 Abbreviations: AS: asthma, AR: allergic rhinitis, CI: confidence interval, DM: diabetes mellitus 